Document downloaded from:

http://hdl.handle.net/10251/155398

This paper must be cited as:

Boruntea, C.; Sastre Navarro, Gl.; Lundegaard, LF.; Corma Canós, A.; Vennestrom, PNR. (2019). Synthesis of High-Silica Erionite Driven by Computational Screening of Hypothetical Zeolites. Chemistry of Materials. 31(22):9268-9276.

https://doi.org/10.1021/acs.chemmater.9b01229

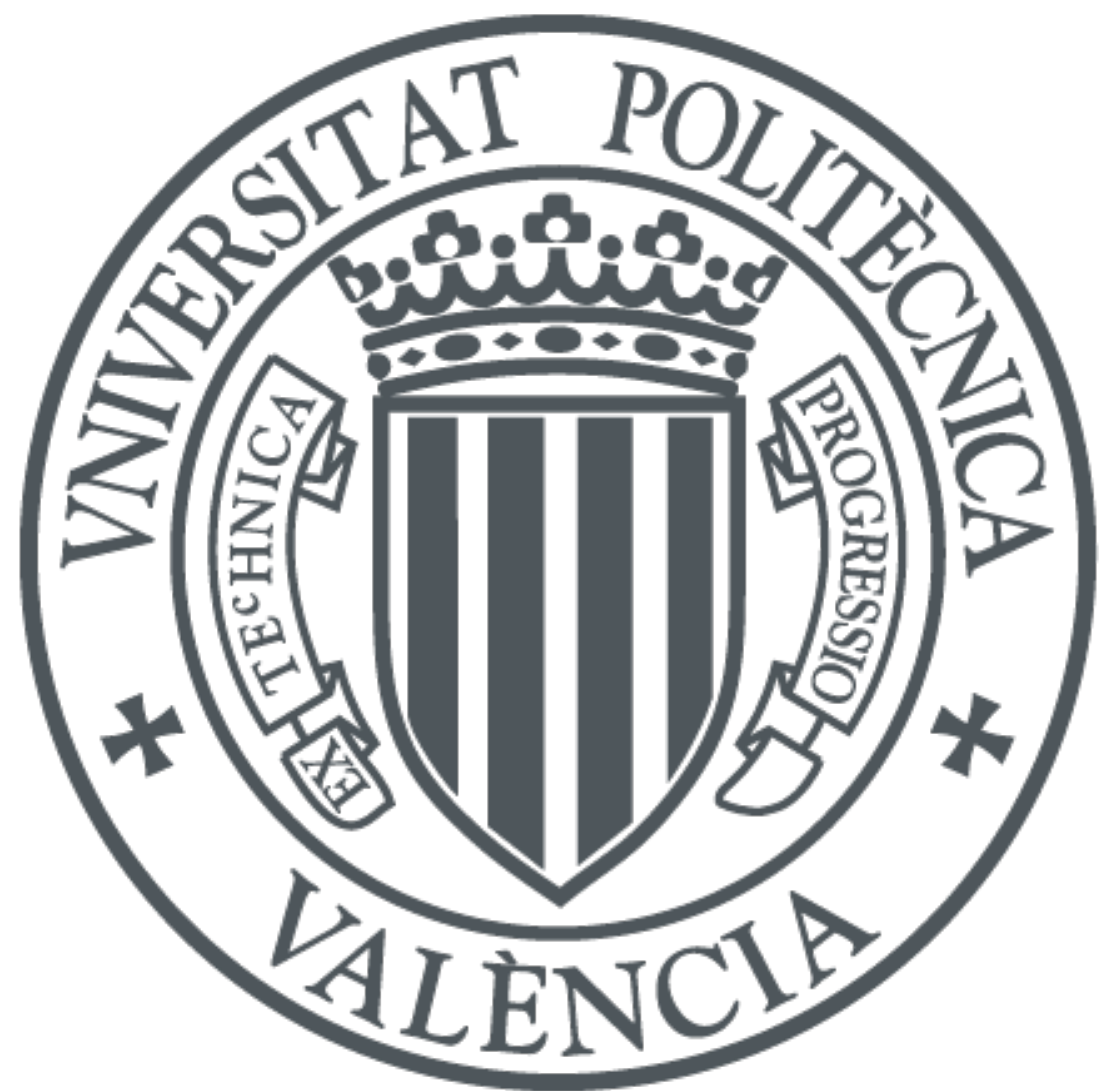

The final publication is available at

https://doi.org/10.1021/acs.chemmater.9b01229

Copyright American Chemical Society

Additional Information

This document is the Accepted Manuscript version of a Published Work that appeared in final form in Chemistry of Materials, copyright (C) American Chemical Society after peer review and technical editing by the publisher. To access the final edited and published work see https://doi.org/10.1021/acs.chemmater.9b01229. 


\title{
Synthesis of high-silica ERI driven by computational screening of hypothetical zeolites
}

\author{
Cristian-R. Boruntea ${ }^{a, b}$, German Sastre ${ }^{b}$, Lars F. Lundegaarda, Avelino Corma ${ }^{b}{ }^{*}$, Peter N. R. Vennestrøm c * \\ a Haldor Topsøe A/S, Haldor Topsøes Alle 1, DK-2800 Kgs. Lyngby, Denmark \\ b Instituto de Tecnología Química, Universitat Politècnica de València-Consejo Superior de Investigaciones \\ Científicas, Avenida de los Naranjos s/n, 46022 Valencia, España \\ c Umicore Denmark ApS, Nøjsomhedsvej 20, DK-2800 Kgs. Lyngby, Denmark
}

\section{Abstract}

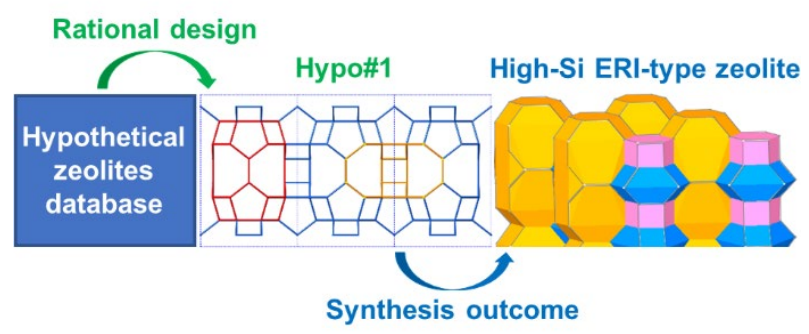

A hypothetical zeolite framework was selected from a database of hypothetical structures and adapted based on structural features relevant for deNOx and MTO catalysis. To attempt the realization of this structure a computational energy-based approach was applied to select relevant OSDAs (organic structure directing agents) molecules with large OSDA-zeolite stabilization energies, leading to the selection of three OSDAs (OSDA1, OSDA2, OSDA3) as potential candidates for the synthesis of the hypothetical zeolite (Hypo\#1). Instead of Hypo\#1, ERI was found to dominate the experimental product outcome when potassium was used as a mineralizing agent. In the case of OSDA3 a novel high-silica ERI was found. The different ERI products were characterized, intergrowth structures ruled out, and special attention was paid to the compositional and morphological features arising from the use of the different OSDAs. In the specific high-Si product obtained using OSDA3 a distinct tubular to prismatic crystal morphology could be seen. Theoretical stabilization energies calculated for potentially competing phases (Hypo\#1, ERI, OFF, CHA among others) could be used to rationalize the experimental outcome to a certain extent, but our results show that considering the zeolite-OSDA interaction is probably not sufficient to realize hypothetical frameworks alone, especially for Al-containing zeolites where alkali ions play an important role during crystallization. 


\section{Introduction}

Zeolites are crystalline microporous aluminosilicate materials composed of tetrahedrally coordinated Tatoms (typically $\mathrm{Si}$ and $\mathrm{Al}$ ), linked together by oxygen bridges. For each tetrahedron with a trivalent element, such as $\left[\mathrm{AlO}_{4 / 2}\right]^{-}$, a negative charge is introduced into the framework that is counterbalanced by a cation. The different ways in which corner connected $\mathrm{SiO}_{4 / 2}$ and $\mathrm{AlO}_{4 / 2}$ tetrahedra are joined together, gives rise to channels, cavities and interconnected voids. The combination of the uniform pore dimensions, ion exchange properties and/or internal acidity combined with thermal stability gives rise to a large number of catalytic applications (1), (2). The development of zeolites as catalyst has historically followed two main tracks namely by: i) exploring known topologies with different compositions, such as Si/Al ratios, heteroatom substitution, and presence of nonframework cations; and ii) discovery of novel topologies with new pore systems, cavity sizes and local coordination environments.

Compositionally, the $\mathrm{Si} / \mathrm{Al}$ ratio is one of the most important parameters that influence zeolite stability and have a direct impact on the catalytic activity. Typically, Si-rich zeolites show improved stability compared to their Al-rich analogs. Contrarily, the amount of aluminum is typically related to the catalytic activity of the zeolite materials and a tradeoff between activity and structural stability is commonly encountered. For many catalytic applications, intermediate $(\mathrm{Si} / \mathrm{Al}=$ ca. $4-10)$ to high-silica $(\mathrm{Si} / \mathrm{Al}>10)$ zeolites are preferred. For this reason, the impact of preparing or discovering novel zeolites becomes much higher when the compositional range can be extended into the intermediate and high-silica range.

Most novel zeolite framework types are discovered through a trial-and-error approach using various families of OSDAs (organic structure directing agents) and chemical intuition. Recently, new rational methods have been proposed for enlarging the list of catalytically relevant zeolite frameworks, by either employing computational methods or by using different synthesis procedures (3), (4), (5), (6), (7). An ab initio zeolite synthesis methodology for targeted applications has also recently been proposed where the OSDAs is chosen to mimic the transition state of a specific reaction following a molecular-recognition pattern (8), (9). Although several databases of hypothetical frameworks have been proposed (10), (11), (12), (13) their practical preparation remains an open question due to the poor understanding of nucleation and crystallization mechanisms. Even if considerable advancement has been made in finding novel OSDAs by computer modeling for already made zeolites (14), (15) this approach seems to be less successful when hypothetical frameworks are targeted.

In this study we explore a rational approach for the preparation of novel zeolite materials, and in this case, targeted towards small-pore zeolite applications such as deNOx or MTO reactions. First, we search for hypothetical frameworks, by imposing certain framework descriptors on a hypothetical zeolite database, followed by finding suitable OSDAs for the selected framework. By experimentally using the computationally predicted OSDAs under hydrothermal conditions, we noticed that another zeolite emerged, namely a novel high-silica version of the ERI framework that competes favorably with the desired hypothetical structure. Results herein are 
rationalized by considering theoretical stabilization energies between different OSDAs, where the synthesis of other possible competing zeolite frameworks are studied for comparison.

\section{Experimental}

\subsection{Computational models and methods}

A framework selection was made from the hypothetical silver database developed by Foster and Treacy (10), (11) containing frameworks with maximum 6 different T-atoms giving a total of 933611 structures, although a large number can be initially discarded by having too high energy ( $>30 \mathrm{~kJ} / \mathrm{SiO}_{2}$ ) leading to 16317 structures from which approximately $50 \%$ contain 3 -rings and can thus also be discarded. The selection was made using the zeoTsites code (16) imposing various criteria believed to be particularly relevant for small-pore catalytic applications (as described in the Results), this leading to the choice of a hypothetical zeolite that we named Hypo\#1 herein. To find suited OSDAs for this hypothetical zeolite, we initially considered OSDAs for cages similar in size and configuration as the hypo\#1 cage. Two overall OSDA families were selected. One family was based on a central flexible alkyl chain and another family was based on a rigid aromatic or aliphatic ring. A number of manual permutations were then considered by introducing substituents and/or functional groups to the primitive structures. The stabilization energy was calculated by applying a molecular docking approach using the COMPASS2 and UFF forcefields available in Materials Studio 8.0 (17). Finally three OSDAs were selected for experimental screening.

A detailed analysis of the stabilization of selected OSDAs together with selected frameworks was later carried out in a similar manner as earlier studies (18), (19). In general, only the zeolite-OSDA van der Waals interaction was taken into account for addressing feasibility. Atomic charges were assigned in the OSDA molecules with the restraint of giving an overall neutral charge. This leads to the simplification of considering only the pure silica framework composition and hence the location of $\mathrm{Al}$ and its preferential interaction with the positive charge of the OSDAs is neglected. We reckon this is a simplification given the important role of the $\mathrm{Al}$ location, as recently demonstrated in computational studies (20), (21). Our computational approach relates better to systems without $\mathrm{Al}$ (pure silica zeolites), but has allowed us to neglect the presence of the counteranion of the OSDA. In fluoride media the counteranion $\left(\mathrm{F}^{-}\right)$can be modelled accurately, but in $\mathrm{OH}^{-}$media defects are often difficult to introduce in a computational model since they are not easy to characterize. In spite of simplifications, models can be very successful if they capture the main interactions at play. This is the case of recent work in cage based zeolites, where the zeolite-OSDA van der Waals interactions play a dominant role and the synthesis is driven towards the zeolite corresponding to larger zeolite-OSDA van der Waals interactions (22), (23). For instance, tetramethylammonium was shown to favor the gme cage while bisdiazabicyclooctane derivatives favored aft and $t$-sfw cages, present in zeolites with AFX and SFW topologies.

For the calculation of the energies, four force fields were considered, dealing with the zeolite, OSDA, zeolite-OSDA and OSDA-OSDA interactions. As said above, the simplification of not considering the Al location leads us to simulate neutralised OSDA molecules (with zero total charge but atomic charges different from zero) and, as a consequence the usual cation-cation OSDA-OSDA repulsion is not properly accounted for in our 
simplified description. Our approach, although including both electrostatic and van der Waals OSDA-OSDA interactions, underestimate the electrostatic OSDA-OSDA repulsion. Because of this, the resulting OSDA-OSDA term is not relevant, and therefore is not included in the discussion. For the zeolite, the recently parameterized rigid-ion force field for silico-alumino-phosphate zeotypes was employed (24), which for the pure silica case is equivalent to a former, less general, parameterization (25). For the zeolite-OSDA, the force field by Catlow et al. (26) was employed, for the OSDA-OSDA interaction, the all-atom OPLS force field (27) was used, and for the internal OSDA the forcefield by Oie et al. (28) was used. One unit cell of zeolite was considered in each case.

In a first set of calculations (set A in Table 2), one molecule of OSDA was introduced and geometry optimized taking into account all the coordinates and cell parameters, as well as all the energetic terms including the electrostatic part through the Ewald summation. The cutoff chosen for the van der Waals interactions was $12 \AA$. From the optimized geometry, it was possible to find the micropores where the OSDA molecules remain occluded in their lowest energy configuration (Table S1). Then, with the optimized geometry, a single point energy calculation was performed to extract the contribution of the van der Waals zeolite-OSDA energy corresponding to one cavity (A values in Table 2). ' $A$ ' values are important since they reflect the contribution of a single zeolite-OSDA interaction and allow you to compare the fit of a single OSDA molecule between various frameworks and could also be related to the early stages of nucleation.

A second set of calculations (set B in Table 2) was performed by filling all cavities and channels with OSDA molecules until the micropore system is fully packed with OSDA molecules, which is typically the situation experimentally found in the synthesis of zeolites. The full van der Waals zeolite-OSDA energy is then divided by the number of $\mathrm{SiO}_{2}$ units in the unit cell ( $\mathrm{B}$ values in Table 2), which allows to extract conclusions about the final system. The $\mathrm{B}$-values are considerable smaller than the A-values by being divided by the number of $\mathrm{SiO}_{2}$ units in the cell, which allows comparisons between different OSDAs for a given zeolite (vertical comparison in Table 2) and also for a given OSDA with different zeolites (horizontal comparison in Table 2).

In summary, both 'set $A$ ' and 'set B' calculations represent zeo-OSDA van der Waals interaction energies. The difference is that 'set A' correspond to only one OSDA molecule per unit cell and the value given is the energy per OSDA molecule, while in the case of 'set B' the unit cell has been saturated with OSDA molecules, and the value given is the zeo-OSDA van der Waals energy of all OSDA molecules divided by the number of $\mathrm{SiO}_{2}$ in the unit cell. All energy minimizations have been carried out allowing the full relaxation of all the atoms in the system as well as the cell parameters using the BFGS (Broyden-Fletcher-Goldfarb-Shanno) (29), (30), (31), (32) within the GULP software (33), (34).

\subsection{Hydrothermal synthesis}

For the attempt to synthesize the Hypo\#1 zeolite, three different approaches were followed. Each OSDA (OSDA1-3) was explored in alkaline conditions with $\mathrm{KOH}$ (set 1) and $\mathrm{NaOH}$ (set 2) by using either a FAU zeolite or co-precipitated amorphous-silica-alumina (ASA) as the T-atom source. Additionally, fluoride conditions were also applied with TEOS and Aluminum isopropoxide as the source of T-atom (set 3). In a typical synthesis 
using FAU as the T-atom source and OSDA 3, a mixture of $1.99 \mathrm{~g}$ cyclohexane-1,4-bis(trimethylammoni um) dihydroxide (12.7 wt. \% aqueous solution), $1.81 \mathrm{~g} \mathrm{KOH}$ (10 wt. \% aqueous solution), $0.45 \mathrm{~g}$ distilled water and $0.74 \mathrm{~g} \mathrm{FAU}$ zeolite $(\mathrm{Si} / \mathrm{Al}=15)$ was prepared. The gel was mixed $1 \mathrm{~h}$ at room temperature and was transferred to a Teflon lined autoclave and heated at $135{ }^{\circ} \mathrm{C}$ for 7 days and the solid product separated by filtration and washing with deionized water. Crystallization was carried out at $135^{\circ} \mathrm{C}$ for seven days unless otherwise stated.. The raw materials used were: butane-1,4-bis(trimethylammonium) dihydroxide (OSDA1), pentane-1,4bis(trimethylammonium) dihydroxide (OSDA2), cyclohexane-1,4-bis(trimethylammonium) dihydroxide (OSDA3), $\mathrm{KOH}$ (reagent grade $\geq 98 \%$, Sigma Aldrich), Y-zeolite (CBV712, CBV720, CBV760 obtained from Zeolyst International), ASA (amorphous-silica-alumina) ( $\mathrm{Si} / \mathrm{Al}=6, \mathrm{Si} / \mathrm{Al}=15$ and $\mathrm{Si} / \mathrm{Al}=30)$ prepared in-house. Detailed synthesis procedures for OSDAs can be found in the Supporting Information.

\subsection{Characterization}

The resulting zeolite materials were analyzed in the as-synthesized form by X-ray diffraction using a Phillips PW1800 instrument system in $\theta-2 \theta$ geometry working in reflectance mode using CuKa radiation $(\lambda=$ $1.541 \AA$ ). SEM images were recorded using an XL30-SEM instrument operated at $10 \mathrm{kV}$. The samples were sprinkled over carbon tape and attached on Al-stubs and then coated with a ca. $30 \mathrm{~nm}$ layer of carbon to prevent charging. TGA-DSC analysis were performed using a Mettler TGA-DSC instrument with a heating rate of $5{ }^{\circ} \mathrm{C} /$ min and the temperature limit at $550^{\circ} \mathrm{C}$. Product composition was determined using inductively coupled plasma optical emission spectroscopy (ICPOES) on acid digested samples using a Perkin Elmer Optima 7300 DV instrument. The ${ }^{27} \mathrm{Al}$ MAS NMR spectra were recorded using a Bruker Avance II HD $400 \mathrm{Mhz}$ instrument at spinning rate of $20 \mathrm{kHz}$ in a Bruker $3.2 \mathrm{~mm}$ probe, using a flip angle of $\pi / 12$ and a recycle delay of $1 \mathrm{~s}$. The ${ }^{29} \mathrm{Si}$ MAS NMR spectra were recorded at spinning rate of $5 \mathrm{kHz}$ in Bruker $4 \mathrm{~mm}$ probe, using a flip angle of $60^{\circ}$ and a recycle delay of $60 \mathrm{~s}$.

\section{Results and Discussion}

A first selection of small-pore frameworks was carried out by imposing an energy requirement that refines only structures with energies lower than (or equal to) $0.15 \mathrm{eV}$ in combination with an 8-ring window requirement. The selection was further limited to 3-dimensional channel systems, only having 4, 5, 6 and 8-rings present in the structure, as well as minimum $80 \%$ of the 8 -rings to be circular. Furthermore, the selected structures were also limited to satisfy feasibility criteria reported elsewhere (35), (36). One framework was selected (entry 139_4_48475) containing 4 T-atoms and $128 \mathrm{SiO}_{2}$ per unit cell. The structure was then modified manually to a simpler structure with less cage diversity. The outcome, named Hypo\#1 herein, contain two different T-atoms (with a multiplicity of 32 and 16 ) and a total of 48 T-atoms ( $\mathrm{SiO}_{2}$ units) per unit cell (see file generated by zeoTsites in the $\mathrm{SI}$ ). Figure 1 shows the framework structure of Hypo\#1, seen along the $\mathrm{a}, \mathrm{b}$ and c directions. 

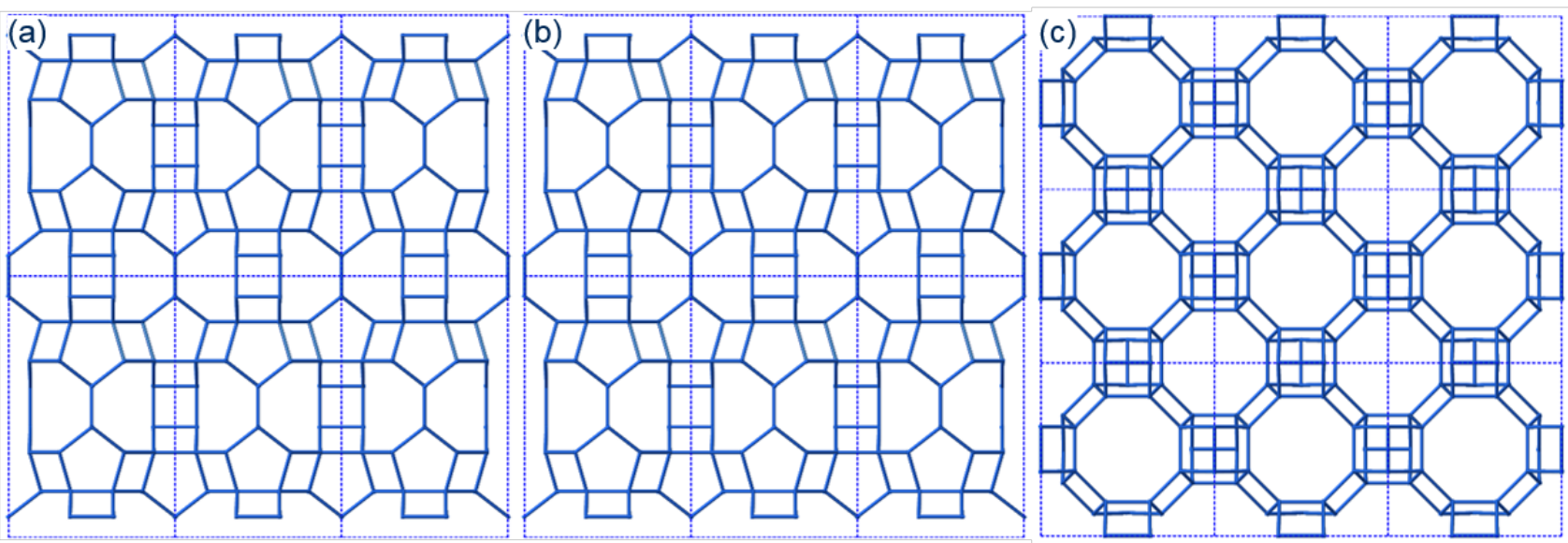

Figure 1 Framework structure of Hypo\#1 zeolite seen along a, b and c direction (blue ribbons represent Si-Si connections. $\mathrm{O}$ atoms are omitted for simplicity)

As a starting point OSDA candidates were selected based on molecules known to stabilize similar sized cages in zeolites where-after an iterative process of manual permutations of substituents was applied. A final selection of three OSDAs (see Figure 2 and Table 2) was afterwards made by taking into account the feasibility of the organic synthesis procedure.

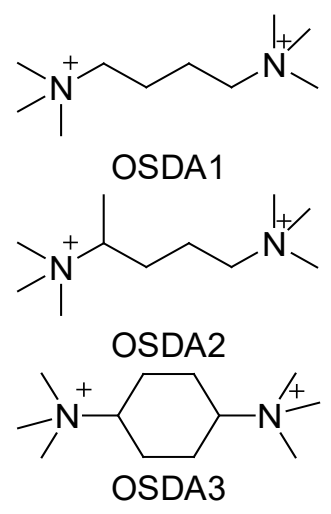

Figure 2 OSDA candidates selected for exploration in hydrothermal synthesis.

To evaluate the potential of the selected OSDAs an extensive experimental evaluation under various conditions and using various raw materials was undertaken. Hydrothermal synthesis was explored using OSDA13 under alkali conditions using either $\mathrm{NaOH}$ or $\mathrm{KOH}$ as the mineralizing agent in combination with either amorphous silica-alumina (ASA) or using a FAU zeolite as the T-atom source. The overall synthesis composition was varied through the Si/Al ratios. Furthermore, crystallization in fluoride media using OSDA1-3 was also investigated as this approach often leads to products strongly dictated by the OSDA-zeolite interaction, especially under Si-rich conditions. 
Crystallization in fluoride media revealed amorphous products together with the presence of an unknown phase (not Hypo\#1 target framework) for OSDA1, independently of the reaction time. For OSDA2 and 3 a mixture of amorphous, LEV, SOD and pure silica AST was obtained as well as an unknown phase (not Hypo\#1 target). The results of the synthesis are reported in the supporting information (Tables S4-S7). The combination of the three OSDAs and $\mathrm{NaOH}$ in general leads to products like ${ }^{*} \mathrm{BEA}$, amorphous or small amounts of an unknown phase (again, not the Hypo\#1 target zeolite); indicating a low specificity of the OSDA-zeolite interaction in this system.

Interestingly, when the mineralizing agent is switched to $\mathrm{KOH}$, we observe either amorphous products or ERI for all three OSDAs. This observation points to a strong influence of potassium on the product selectivity in combination with the stabilizing effect of the various OSDA molecules. Table 1 summarizes the products obtained from the synthesis with ASA and FAU as sole Si and Al source for the $\mathrm{KOH}$ system.

Erionite (ERI) is a well-known small-pore zeolite, that can be found in nature with a Si/Al ratio around 3 (37). It is typically seen as an intergrowth with Offretite (OFF) (38). Pure-phase ERI with higher Si/Al ratios was first reported by Hong et al. by combination of tetraethylammonium, linear diquaternary alkylammonium ions as OSDAs and a charge density mismatch (CDM) approach (39), (40). The UZM-12 material crystallizes mainly in the presence of $\mathrm{K}^{+}$or $\mathrm{Rb}^{+}$ions and the highest $\mathrm{Si} / \mathrm{Al}$ ratio achieved has been 6.5. Recently, and yet only reported in the patent literature, is the preparation of SSZ-98, a high-silica ERI molecular sieve with either rod-like or discshaped crystal morphologies. Using N,N'-dimethyl-1,4-diazobicyclo[2.2.2]octane as the OSDA (OSDA4, Table 2), Si/Al ratios as high as 13.5 were reported for SSZ-98 (41).

Table 1 Products and chemical composition of selected synthesis using OSDA1, OSDA2 and OSDA3 from synthesis composition $1.0 \mathrm{SiO}_{2}$ : x Al : 0.1 OSDA : $0.3 \mathrm{KOH}: 20 \mathrm{H}_{2} \mathrm{O}$ crystallized for 7 days at $135^{\circ} \mathrm{C} .(\mathrm{x}=0.167,0.066$ and 0.033 )

\begin{tabular}{cccccc}
$\begin{array}{c}\text { Sample } \\
\text { Number }\end{array}$ & OSDA & Si and Al-source & Product & Si/Al & K/Si \\
\hline 1 & 1 & ASA (Si/Al=6) & ERI & $\mathbf{4 . 4}$ & $\mathbf{0 . 1 1}$ \\
2 & 1 & ASA (Si/Al=15) & amorphous & - & - \\
3 & 1 & FAU (Si/Al=6) & ERI & $\mathbf{4 . 9}$ & $\mathbf{0 . 1 1}$ \\
4 & 1 & FAU (Si/Al=15) & amorphous & - & \\
\hline 5 & 2 & ASA (Si/Al=6) & ERI & - & - \\
6 & 2 & ASA (Si/Al=15) & amorphous & - & $\mathbf{0 . 1 0}$ \\
7 & 2 & FAU (Si/Al=6) & ERI & $\mathbf{5 . 1}$ & - \\
8 & 2 & FAU (Si/Al=15) & amorphous & - & - \\
\hline 9 & 3 & ASA (Si/Al=6) & ERI & - & $\mathbf{0 . 1 7}$ \\
10 & 3 & ASA (Si/Al=15) & ERI & - & $\mathbf{0 . 1 1}$ \\
11 & 3 & FAU (Si/Al=6) & ERI & $\mathbf{4 . 9}$ & \\
12 & 3 & FAU (Si/Al=15) & ERI & $\mathbf{1 1 . 0}$ &
\end{tabular}


For all OSDAs ERI is observed at a Si/Al ratio of 6 in the synthesis gel when $\mathrm{KOH}$ is used. However, at higher $\mathrm{Si} / \mathrm{Al}$ ratios ERI is only obtained using OSDA3. The $\mathrm{Si} / \mathrm{Al}$ measured in this product is 11 , which is the highest $\mathrm{Si} / \mathrm{Al}$ ratio reported for ERI in the scientific literature so far. The observation that ERI is obtained even at higher Si/Al ratios using OSDA3 indicates that it does indeed provide a suitable structure directing effect for this particular framework topology.
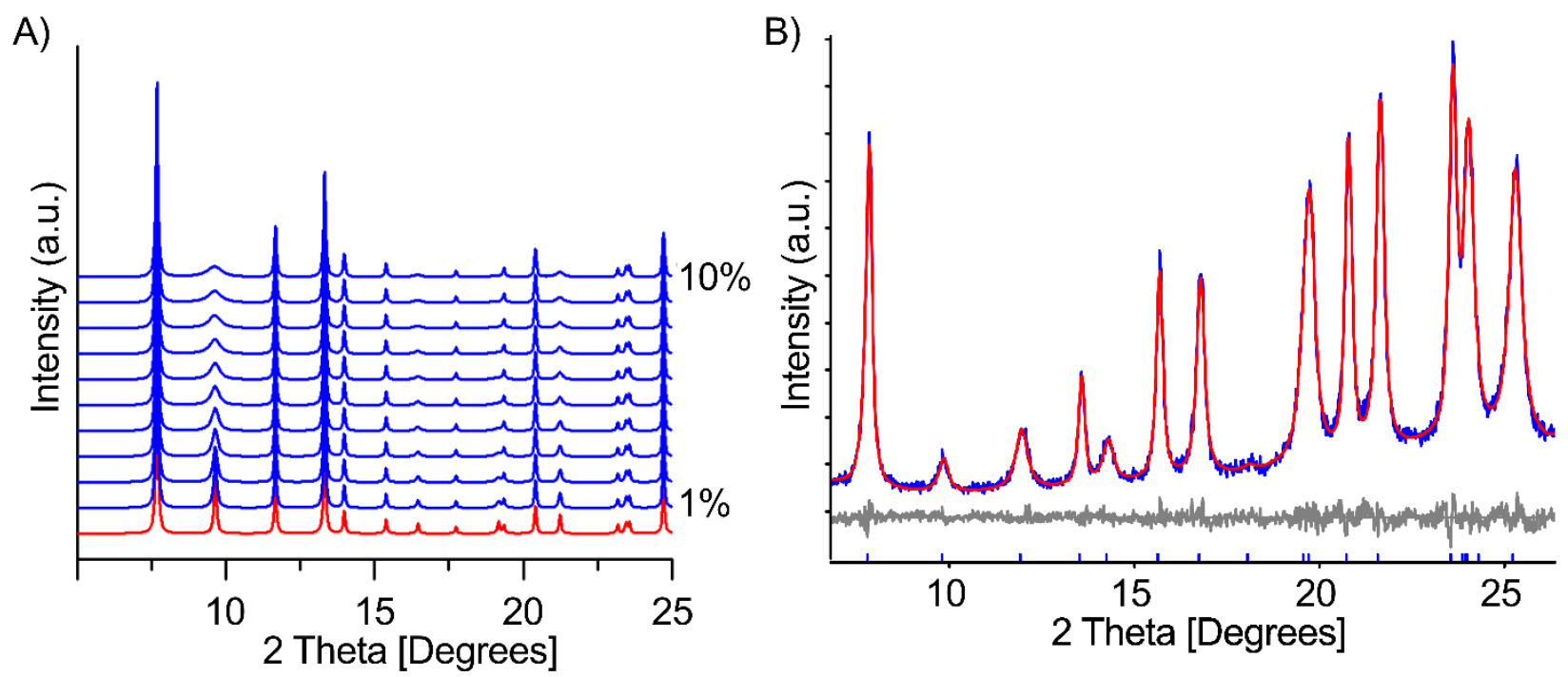

Figure 3 XRPD patterns of ERI. A) Simulated patterns for ERI (100\% ERI and $0 \%$ OFF, red curve) and for ERI-OFF intergrowth (1\% OFF up to $10 \%$ OFF, blue curves). B) Observed pattern of sample 12 (blue curve) and a model curve (Red) obtained using a LeBail model including peak shape parameters related to particle average size and shape. The grey curve is the difference between the model and the observed data.

It is well-known that ERI typically crystalizes with intergrowths of OFF. Figure $3 \mathrm{~A}$ shows simulated PXRD patterns for the $100 \%$ ERI-type zeolite and for the ERI/OFF intergrowths, with various percentages of OFF $(1-10 \%)$. Already at low amounts of intergrowths anisotropic broadening is seen for some of the peaks with increasing amounts of intergrowth. The observed PXRD pattern of sample 12 does not show additional broadening of the peak at 9 degrees 2 theta, and therefore no indication of stacking disorder in the sample. The difference in relative peak intensities in figure $3 \mathrm{~A}$ and $3 \mathrm{~B}$ respectively, is mainly a result of collecting the observed data using variable divergence slits. X-ray diffraction patterns for all obtained ERI products are shown in Figure 4. Only small differences are seen within the synthesized samples, and XRD peak broadening features related to intergrowth with OFF are not observed in any of the observed data. 


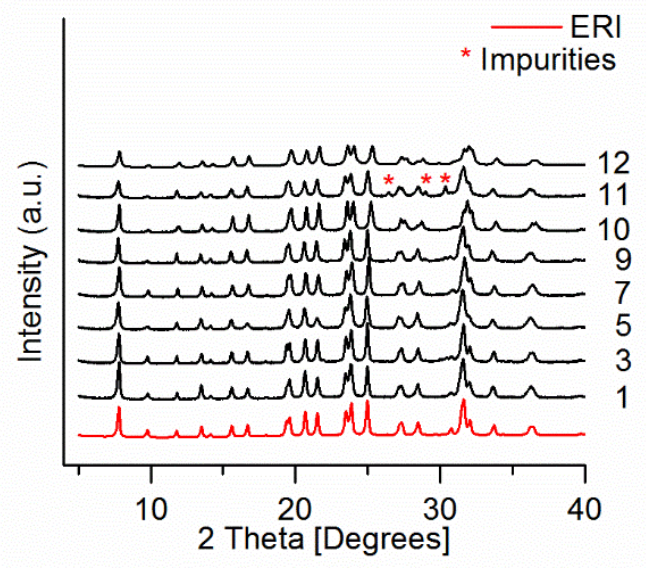

Figure 4 XRPD patterns of as-synthesized ERI-type zeolite products using OSDA1 (samples 1 and 3), OSDA2 (sample 5 and 7 ) and OSDA3 (samples 9,10,11,12). The bottom red pattern shows the calculated pattern for ERI.

$\mathrm{ERI}$ is obtained from both amorphous starting materials and the crystalline FAU. Indicating that the reactivity or dissolution rate of the starting material may not be critical for the achievement of ERI as it was observed e.g. for AEI and AFX (42). Synthesis of intermediate-silica (Si/AI = 6.5, UZM-12) ERI using OSDA1 has so far only been reported using the CDM-approach. Whether the use of co-precipitated silica-alumina provides an analogous starting point as the initial precipitation of CDM-gels is unclear, but some similarity in reactivity could exist.

In all cases potassium is seen in the final product. A molar $\mathrm{K} / \mathrm{Si}$ ratio of 0.11 and $\mathrm{Si} / \mathrm{Al}$ ratios between 4.4 and 11 corresponds to approximately $3.2-3.6 \mathrm{~K}$ per unit cell that contains 2 can cages, so it is reasonable to assume that most of the can cages are occupied by $\mathrm{K}^{+}$ions and perhaps some $\mathrm{K}^{+}$is also located in the larger eri cages or double-six ring structures also contained in the ERI framework. By comparison of ${ }^{13} \mathrm{C}$ CP-MAS NMR of the solid product with ${ }^{13} \mathrm{C}-\mathrm{NMR}$ of the OSDA in solution before use (see Figure S2) we find that the OSDAs remain intact throughout the crystallization and are indeed successfully occluded within the cavities of the ERI materials. From TGA analysis of OSDA3 combustion, a weight loss of $21.2 \%$ was measured (see Figure S1), which correspond to 3.1 molecules per unit cell or 1.0 OSDA molecules per eri cage, whereas this number is lower for OSDA1 and 2. Altogether this points towards the efficient use of the OSDA molecules with approximately one OSDA molecule per eri cage and $\mathrm{K}$ located both in the can and eri cages, especially for OSDA3.

The ${ }^{29}$ Si MAS NMR measurements for sample 12 (Figure 5a) reveal 3 distinctive peaks that have been assigned to $\mathrm{Si}(0 \mathrm{Al}), \mathrm{Si}(1 \mathrm{Al}), \mathrm{Si}(2 \mathrm{Al})$ and a broad signal which was assigned to $\mathrm{Si}(3 \mathrm{Al})$. Deconvolution and calculation of the Si/Al ratio (10) is in good agreement with that measured from ICP (11). The ${ }^{27} \mathrm{AI}$ MAS NMR spectrum for ERI-type zeolites corresponding to sample 12 (Figure $5 \mathrm{~b}$ ) shows that all the aluminum is located in framework positions with tetrahedral coordination (Alv) (peak at approx. $60 \mathrm{ppm}$ ). 

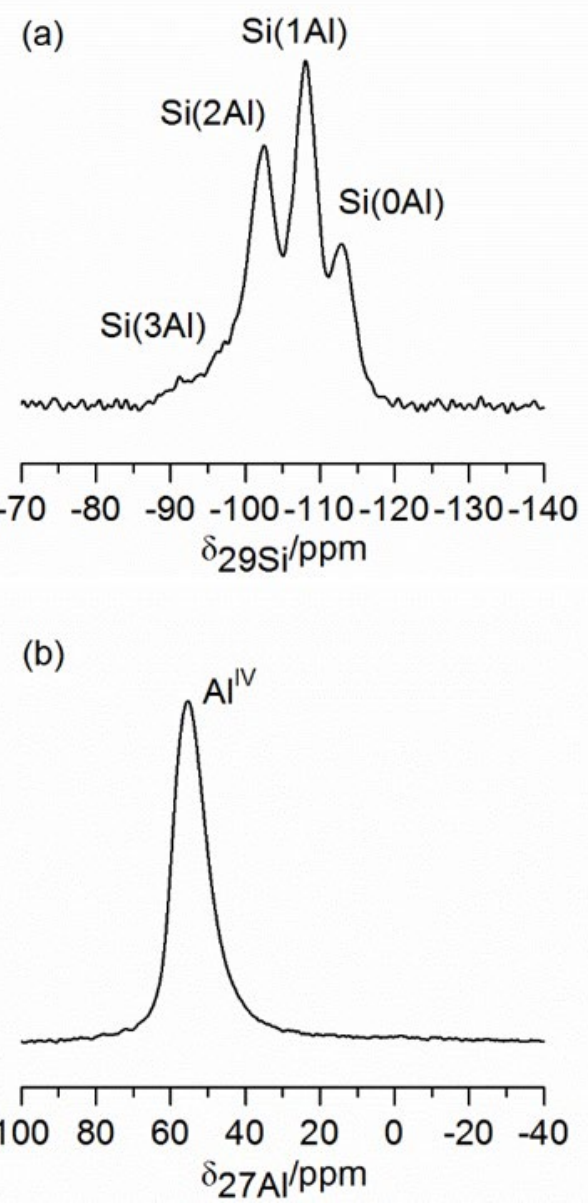

Figure $5{ }^{29} \mathrm{Si}(\mathrm{a})$ and ${ }^{27} \mathrm{Al}$ MAS NMR (b) spectra of ERI-type zeolites corresponding to sample 12. 

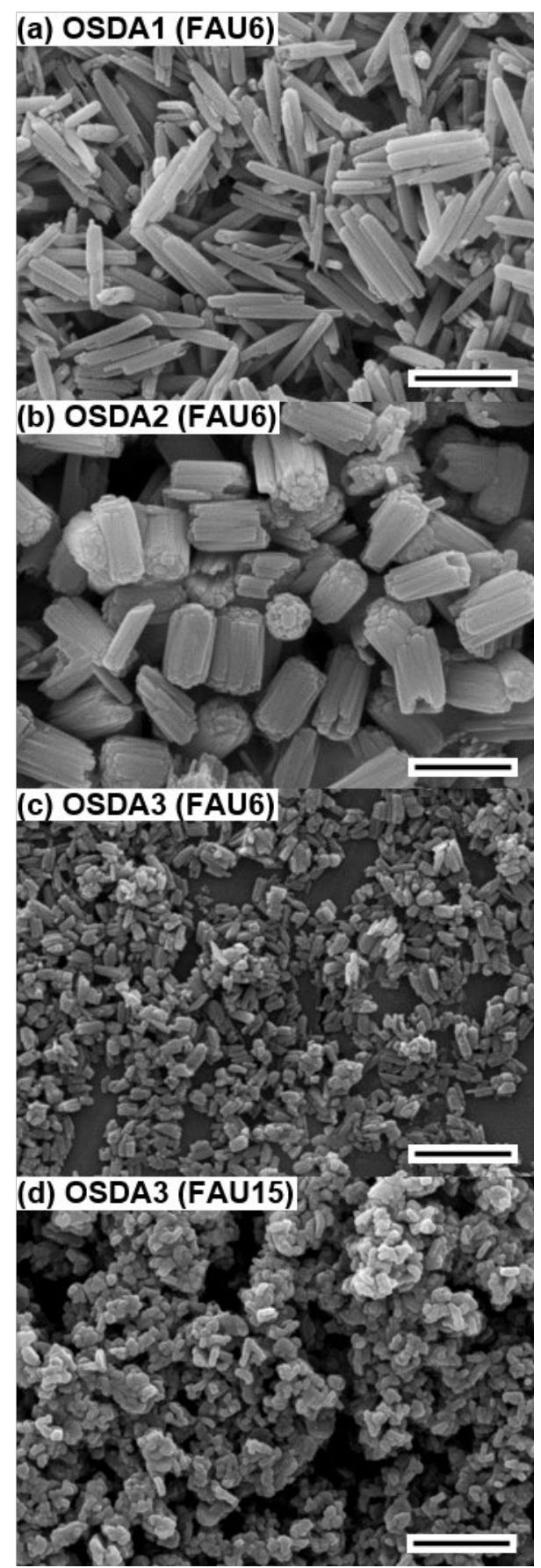

Figure 6 SEM images of ERI materials corresponding to samples 3, 7,11 and 12 respectively, synthesized using FAU as the $\mathrm{Si}$ - and $\mathrm{Al}$-source. Scale bars in the images correspond to $1 \mu \mathrm{m}$.

SEM images of the ERI products show various crystal morphologies. For OSDA1 needles or rods are obtained, whereas for OSDA2 shorter and grouped parallel fibers are seen. OSDA3 gives slightly irregular, in this case smaller, crystallites, with tabular to prismatic crystal morphologies. 
Recently, the influence of the particle morphologies on the performance of the zeolite catalysts has led to important findings. For example Teketel et. al. (43) observed that for the MFS (ZSM-57) particles with a platelet morphology a resistance towards deactivation for the (methanol-to-hydrocarbons) MTH reaction is seen, compared with the SZR (SUZ-4) needle-like crystals, where a rapid deactivation is noticed. Another example is the study made by Lukaszuk et. al. which correlated the product shape selectivity for the MTH reaction with four distinct particle morphologies for OFF-type zeolites (44). As the SEM images in Figure 6 show, control of the morphology can be achieved for ERI by selection of the OSDA, which besides the Si/AI ratio will allow one to modify the catalytic behavior.

\subsection{General considerations in the preparation of Hypo\#1 and ERI zeolites}

To rationalize the experimental results obtained, a thorough investigation of the calculated stabilization energies of the OSDAs with the Hypo\#1, ERI and various known impurity phases was carried out. The OSDAs considered for the synthesis of Hypo\#1 are summarized in

Table 2. As described earlier, the main experimental phase observed from the various OSDAs was ERI with no evidence of Hypo\#1. CHA and LTL frameworks are also well known impurities during synthesis of ERI zeolites (45) and dense phases such as e.g. PHI, SOD, GIS can also appear. For comparison we have also included the reported OSDA (OSDA4) for SSZ-98, which also experimentally gives the high-Si ERI.

The A values in Table 2 correspond to energies that can be compared across different zeolites for the same OSDA (horizontal comparison in Table 2), but they should not be taken as giving the most stable zeolite system since only one cavity in the unit cell (and not the full micropore system) is considered. B values on the other hand take into account an optimum full loading and thus provides a more realistic prediction of synthesis feasibility. One should be cautious in comparing B-values only as OSDA-OSDA interactions will also be reflected in this number, which is the case when more than one OSDA occupies each cavity. Each set of values is complementary and both should be considered when addressing the stabilization of a framework.

From the theoretical stabilization energies reported in Table 2 (A values) the selected OSDAs should favor Hypo\#1 and ERI. This indicates that there is a good fit between the OSDAs and the cages in ERI and Hypo\#1, respectively. Interestingly, the A value for OSDA4 in ERI is not as high as OSDA1-3 suggesting that a single OSDA4 is not sufficient to stabilize the cage in ERI. When full loadings are considered (Table 2, B values) the picture changes. Now CHA and ERI are favored phases instead of Hypo\#1, and this could indeed be one of the reasons why Hypo\#1 is not achieved. On the other hand, CHA was never achieved either, again pointing toward a specific behavior of the potassium, which is not captured in the theoretical description.

To elaborate further on the achievement of ERI, and the expansion of the Si/Al ratio especially from OSDA3, it is useful to consider the flexibility of the various OSDAs, as different confirmations typically provides less specificity. OSDA1 was previously reported for the synthesis of UZM-12 (ERI framework) and has a high degree of flexibility due to the hydrocarbon chain linking the two trimethylammonium ends. This allows a large 
number of stabilized conformational configurations. The length of this molecule shows a good fit with the eri cage since it adopts an all-trans configuration when located inside the cage, whereas when the molecule is extended e.g. by increasing the hydrocarbon chain length or by substituting end-methyl groups with ethyl groups a gauche conformation is adopted (39).

For OSDA2, the flexibility is lower due to the presence of the methyl group attached to the hydrocarbon chain, which introduces a higher barrier for rotation around the tertiary $\mathrm{sp}^{3}$ hybridized carbon atom. The number of conformations are therefore limited, and it is less likely to curl together and adopt too many conformations.

In OSDA3, the hydrocarbon backbone is replaced with a cyclohexane ring. The ring-structure introduces more rigidity into the molecule. Nevertheless, conformations such as a boat, chair or a twist form are still possible. Furthermore, the length of OSDA3 is comparable to OSDA1 and OSDA2 (see Table 2). Common for OSDA1-3 is that one molecule occupies each eri cage.

In comparison SSZ-98, which also has the ERI structure, and has recently been reported in the patent literature uses N,N'-dimethyl-1,4-diazobicyclo[2.2.2]octane (OSDA4) (41). This molecule shows an even higher degree of rigidity, but does not have the same length.

Table 2 Characteristics of OSDAs and their stabilization energies (energies are expressed in $\mathrm{kJ}$ for $\mathrm{A}$ values and $\mathrm{kJ} / \mathrm{SiO} \mathrm{for}_{2}$ $B$ values) for Hypo\#1, ERI, CHA and OFF. OSDAs 1-3 were prepared in this work, while OSDA4 has recently been used to synthesize SSZ-98 (ERI) reported in the patent literature (41).

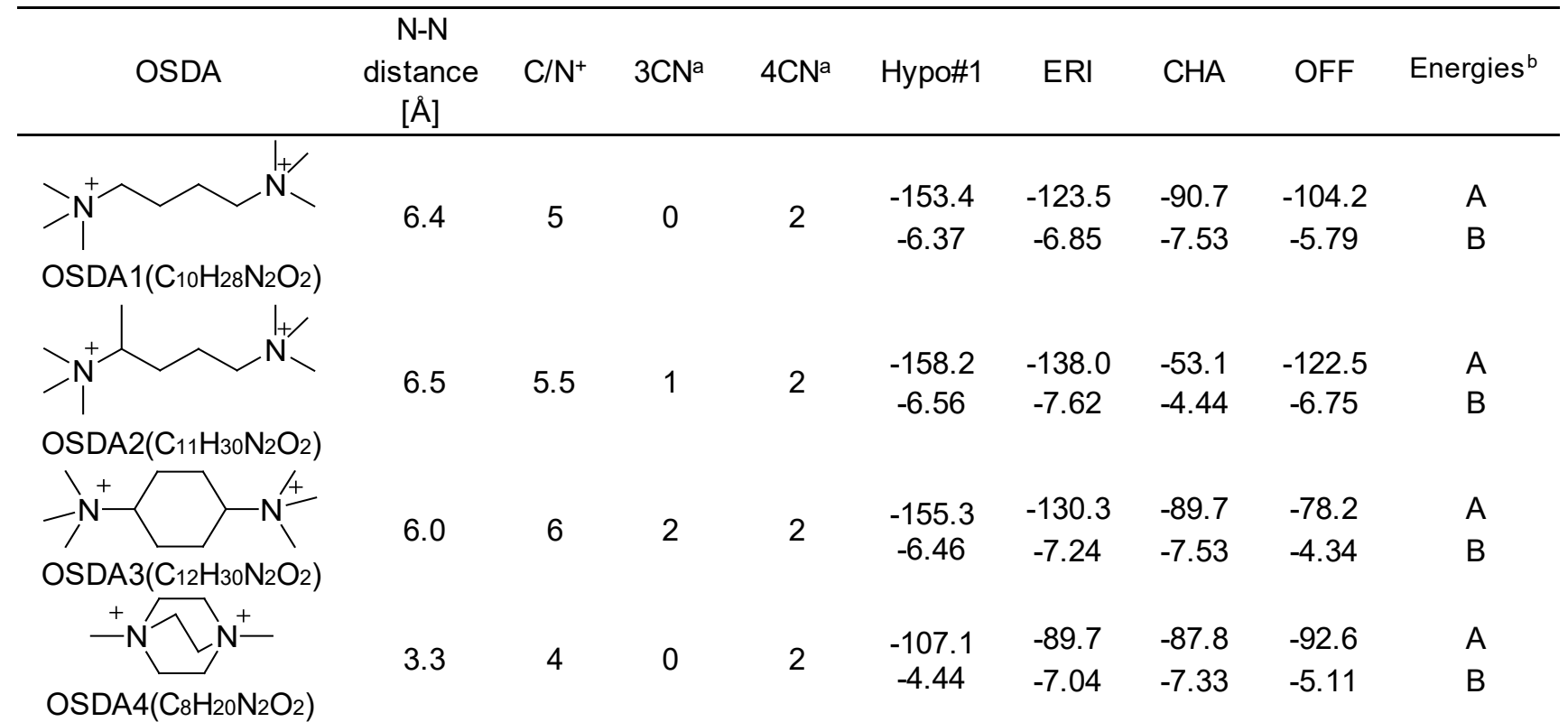

a Number of tertiary $(3 \mathrm{CN})$ and quaternary $(4 \mathrm{CN})$ carbon and nitrogen atoms in the OSDA as defined in (47).

${ }^{b}$ zeolite-OSDA van der Waals energycorresponding to: single occupation (A), full occupation divided by \#SiO 2 (B). For each framework the unit cells contain the following number of $\mathrm{SiO}_{2}$ units: 48 (Hypo\#1), 36 (ERI), 36 (CHA), 36 (OFF). Data for set B: in Hypo\#1 and OFF, all OSDAs are in a loading of 2 OSDAs per unit cell. In ERI, OSDA1, OSDA2, OSDA3 are in a loading of 2 OSDAs per unit cell, while OSDA4 is in a loading of 4 OSDAs per unit cell. In CHA all OSDAs are in a loading of 3 OSDAs perunit cell. 
A quantitative way of expressing the rigidity of an OSDA is by considering the number of tertiary and quaternary carbons and nitrogen (47). These values are reported in Table 2 and are in good agreement with the qualitative description of rigidity given above. The observation of a higher rigidity of OSDA3 and OSDA4 seems to be in good agreement with the fact that these two molecules leads to higher Si/Al products.

Besides rigidity and length, the hydrophobicity of the OSDA is important for the OSDA-framework interaction especially during crystallization. Kubota et al. report that the $\mathrm{C} / \mathrm{N}^{+}$ratio is a good estimate, and that OSDAs with values between 10 and 16 are particularly useful for obtaining high-silica zeolites (47). All the OSDAs used in this work fall outside this range due to the dicationic nature and OSDA3 gives the highest number; perhaps indicating that even more Si-rich materials are achievable with this OSDA.

The theoretical stabilization energy for OSDA4 in ERI is comparable to the numbers we find for OSDA1-3 despite the shorter molecular length. This is in good agreement with the observation that for all other B-values calculated a full loading is achieved with one OSDA per cage, whereas for OSDA4 a full loading in ERI is achieved with two OSDA molecules per cage. Specifically for this OSDA the favorable stabilization energy is achieved with two of the OSDA4 molecules located perpendicular to each other in the eri cage. The most stable conformations of the OSDA1-4 inside the eri cage are shown in Figure 7.

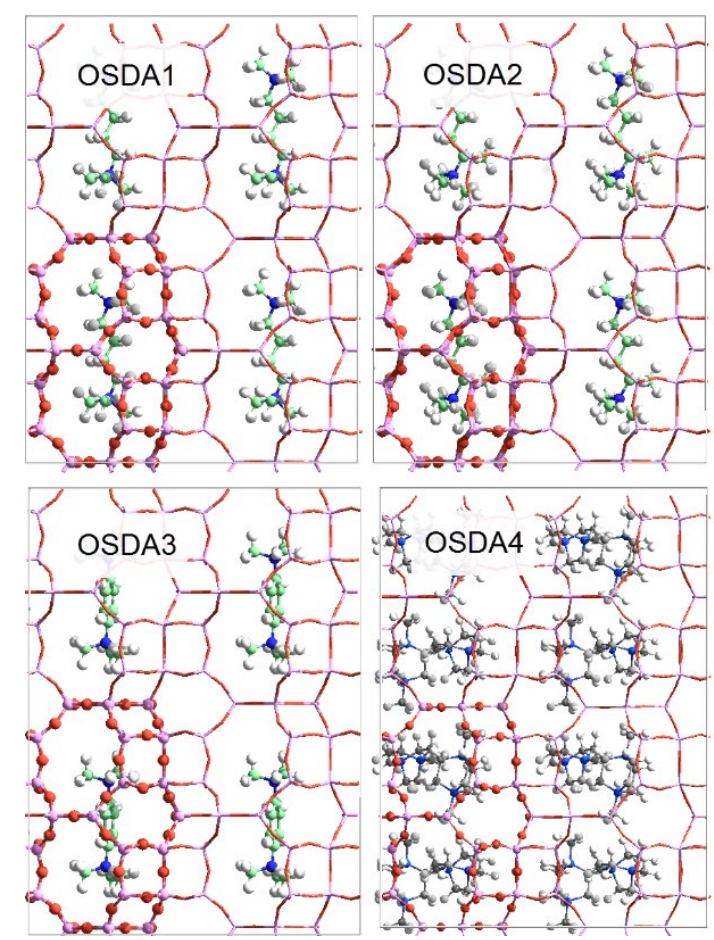

Figure 7 Location and configuration of the OSDAs inside the eri cage (highlighted). Neighbor $(2 \times 1 \times 2)$ unit cells are shown for the sake of clarity. The view shown is perpendicular to the $b$ axis.

\section{Conclusions}


In this work we have investigated the peculiar problem of synthesizing hypothetical zeolite frameworks that have been computationally predicted, but never practically prepared. We have followed a rational approach by screening a database of 933611 structures (www.hypotheticalzeolites.net) and selecting a hypothetical framework. The selected framework was subsequently modified to obtain a more simplified structure, which was designated Hypo\#1. Three different OSDAs were selected based on zeolite-OSDA vdW stabilization energies to attempt the experimental realization of Hypo\#1. When screening the experimental outcome under fluoride and alkali $(\mathrm{NaOH}$ and $\mathrm{KOH})$ hydrothermal conditions the target zeolite was never obtained. Instead we noticed that in the presence of potassium, the synthesis product was ERI, and for OSDA3 a high-Si version could be achieved. In spite of not obtaining a new framework, the morphological features and high Si composition of the obtained ERI-zeolites show promising features for improving various catalytic applications.

Stabilization energies of OSDAs in Hypo\#1, ERI, as well as other zeolite frameworks were calculated to rationalize the results. All tested OSDAs (1-3) provides a good fit for the cage in Hypo\#1 when one OSDA is considered (A-values, Table 2). However, when an optimum loading is considered ERI and CHA are favored phases (B-values, Table 2). This can partly explain why ERI is achieved instead of Hypo\#1, but also highlights the limitations of the approach as CHA is not achieved. ERI is known to crystallize with potassium as the mineralizing agent and thus play a role during crystallization, which is not captured by only considering the zeolite-OSDA vdW stabilization energies. The high Si version of ERI achieved with OSDA3 as well as OSDA4 reported for SSZ-98 is similarly not only explained by the stabilization energies, but seem to relate to the rigidity of the molecule as well.

As this study illustrates, it still remains a challenge to synthesize hypothetical frameworks predicted by in silico modeling. One of the barriers is due to the kinetic control that drives zeolite crystallization along with other factors such as the stabilization of the intermediates and the reaction conditions, which are not typically considered in the computational screening. However, the computational modeling of the zeolite-OSDA interaction still provides a viable starting point for the rational design and synthesis of novel zeolites. It especially provides a method for improving and extending the composition of already existing materials, by providing novel OSDAs and by limiting explorative trial-and-error methods.

\section{Acknowledgements}

The authors thank Haldor Topsoe A/S and Innovation Fund Denmark for financial support under the Industrial PhD programme (Case no. 1355-0174B). We thank MINECO of Spain for funding (SEV-2016-0683 and RTI2018-101033-B-100) and ASIC-UPV for the use of computational facilities. We also thank Prof. M. M. J. Treacy for assistance with the Database of Prospective Zeolite Structures.

\section{Supporting information}

The Supporting Information is available free of charge on the ACS Publications website at DOI: [insert DOI].

- Experimental details of OSDA synthesis, products and chemical composition of products obtained using OSDA1-3 under a broad range of crystallization conditions, details on the OSDA location from 
computational optimizations, thermogravimetric and NMR data of prepared OSDAs and ERI zeolites, as well as zeoTsites output generated for the reported Hypo\#1 structure.

\section{References}

(1) Flanigen, E. M. In Introduction to Zeolite Science and Practice; van Bekkum, H., Flanigen, E. M., Jansen, J. C., Eds.; Elsevier Science: Amsterdam, 1991; Chapter 2, pp. 13-34.

(2) Moscou L. In Introduction to Zeolite Science and Practice; van Bekkum, H., Flanigen, E. M., Jansen, J. C., Eds.; Elsevier Science: Amsterdam, 1991; Chapter 1, pp. 1-12.

(3) Itabashi, K.; Kamimura, Y.; lyoki, K.; Shimojima, A.; Okubo, T. A working hypothesis for broadening framework types of zeolites in seed-assisted synthesis without organic structure-directing agent. J.Am. Chem. Soc. 2012, 134, 11542-11549.

(4) Lu, P.; Villaescusa, L. A.; Camblor, M. A. Driving the Crystallization of Zeolites. Chem. Rec. 2018, 18, 1-12.

(5) Guo, P.; Shin, J.; Greenaway, A. G.; Min, J. G.; Su, J.; Choi, H. J.; Liu, L.; Cox P. A.; Hong S. B.; Wright, P. A.; Zou X A zeolite family with expanding structural complexity and embedded isoreticular structures. Nature, 2015, 524, 74-78.

(6) Tsunoji, N.; Yuki, S.; Oumi, Y.; Sekikawa, M.; Sasaki, Y.; Sadakane, M.; Sano, T. Design of Microporous Material HUS-10 with Tunable Hydrophilicity, Molecular Sieving, and $\mathrm{CO}_{2}$ Adsorption Ability Derived from Interlayer Silylation of Layered Silicate HUS-2. ACS Appl. Mater. Interfaces, 2015, 7, 24360-24369.

(7) Verheyen, E.; Joos, L.; Van Havenbergh, K.; Breynaert, E.; Kasian, N.; Gobechiya, E.; Houthoofd, K.; Martineau, C.; Hinterstein, M.; Taulelle, F.; Van Speybroeck, V.; Waroquier, M.; Bals, S.; Van Tendeloo, G.; Kirschhock, C. E. A.; Martens, J. A. Design of zeolite by inverse sigma transformation. Nature Materials, 2012, 11, 1059-1064.

(8) Gallego, E. M.; Portilla, M. T.; Paris, C.; León-Escamilla, A.; Boronat, M.; Moliner, M.; Corma, A. "Ab initio" synthesis of zeolites for preestablished catalytic reactions. Science, 2017, 355, 1051-1054.

(9) $\mathrm{Li}$, C.; Paris, $\quad$ C.; Martínez-Triguero, J.; Boronat, M.; Moliner, M.; Corma, A. Synthesis of reaction-adapted zeolites as methanol-to-olefins catalysts with mimics of reaction intermediates as organic structure-directing agents. Nature Catalysis, 2018, 1, 547-554.

(10) Foster, M. D.; Treacy, M. M. J. A Database of Hypothetical Zeolite Structures. http://www. hypotheticalzeolites.net (accessed March 9, 2015).

(11) Treacy, M. M. J.; Randall, K. H.; Rao, S.; Perry, J. A.; Chadi, D. J. Enumeration of periodic tetrahedral frameworks. Z. Kristallogr. 1997, 212, 768-791.

(12) Delgado-Friedrichs, O.; Dress, A. W. M.; Huson, D. H.; Klinowski, J.; Mackay, A. L. Systematic enumeration of crystalline networks. Nature, 1999, 400, 644-647.

(13) Earl, D. J.; Deem, M. W. Toward a Database of Hypothetical Zeolite Structures. Ind. Eng. Chem. Res. 2006, 45, 5449-5454. 
(14) Davis, T. M.; Liu, A. T.; Lew, C. M.; Xe, D.; Benin, A. I.; Elomari, S.; Zones, S. I.; Deem, M. W. Computationally Guided Synthesis of SSZ-52: A Zeolite for Engine Exhaust Clean-up. Chem. Mater., 2016, 28, 708-711.

(15) Schmidt, J. E.; Deem, M. W., Lew, C.; Davis, T. M. Computationally-Guided Synthesis of the 8-Ring Zeolite AEI. Top. Catal. 2015, 58, 410-415.

(16) Sastre, G.; Gale, J. D. ZeoTsites: a code for topological and crystallographic tetrahedral sites analysis in zeolites and zeotypes. Microporous Mesoporous Mater. 2001, 43, 27-40.

(17) Dassault Systèmes BIOVIA. https://www.3dsbiovia.com (accessed March 16, 2015), Materials Studio, 8.0, San Diego: Dassault Systèmes.

(18) Sastre, G. A computational chemistry insight into the role of structure directing agents in the synthesis of zeolites. Phys. Chem. Chem. Phys. 2007, 9, 1052-1058.

(19) Burton, A. W.; Zones, S. I. Organic molecules in zeolite synthesis: Their preparation and structuredirecting effects. Stud. Surf. Sci. Catal. 2007, 168, 137-179.

(20) Muraoka, K.; Chaikittisilp, W.; Yanaba, Y.; Yoshikawa, T.; Okubo, T. Directing Aluminum Atoms into Energetically Favorable Tetrahedral Sites in a Zeolite Framework by Using Organic Structure-Directing Agents. Angew. Chem. Int. Ed. 2018, 57, 3742.

(21) Muraoka, K.; Chaikittisilp, W.; Okubo, T. Energy Analysis of Aluminosilicate Zeolites with Comprehensive Ranges of Framework Topologies, Chemical Compositions, and Aluminum Distributions. J. Am. Chem. Soc. 2016, 138, 6184-6193.

(22) Turrina, A.; Garcia, R.; Cox, P. A.; Casci, J. L.; Wright, P. A. Retrosynthetic Co-Templating Method for the Preparation of Silicoaluminophosphate Molecular Sieves. Chem. Mater. 2016, 28, 4998-5012.

(23) Turrina, A.; Garcia, R.; Watts, A. E.; Greer, H. F.; Bradley, J.; Zhou, W.; Cox, P. A.; Shannon, M. D.; Mayoral, A.; Casci, J. L.; Wright, P. A. STA-20: An ABC-6 Zeotype Structure Prepared by Co-Templating and Solved via a Hypothetical Structure Database and STEM-ADF Imaging. Chem. Mater. 2017, 29, 2180-2190.

(24) Ghysels, A.; Moors, S. L. C.; Hemelsoet, K.; De Wispelaere, K.; Waroquier, M.; Sastre, G.; Van Speybroeck, V. Shape-Selective Diffusion of Olefins in 8-Ring Solid Acid Microporous Zeolites. J. Phys. Chem. C 2015, 119, 23721-23734.

(25) Bushuev, Y. G.; Sastre, G. Atomistic simulations of water and organic templates occluded during the synthesis of zeolites. Micropor. Mesopor. Mater. 2010, 129, 42-53.

(26) Catlow, C. R. A.; Freeman, C. M.; Vessal, B.; Tomlinson, S. M.; Leslie, M. Molecular dynamics studies of hydrocarbon diffusion in zeolites. J. Chem. Soc. Faraday Trans. 1991, 87, 1947-1950.

(27) Jorgensen, W. L.; Maxwell, D. S.; Tirado-Rives, J. Development and testing of the OPLS all-atom force field on conformational energetics and properties of organic liquids. J. Am. Chem. Soc. 1996, 118, 11225-11236.

(28) Oie, T.; Maggiora, G. M.; Christoffersen, R. E.; Duchamp, D. J.; Development of a flexible intra- and intermolecular empirical potential function for large molecular systems. Int. J. Quantum Chem. Quantum Biol. Symp. 1981, 20, 1-47. 
(29) Broyden, C. G. The convergence of a class of double-rank minimization algorithms. J. Inst. Math. Appl. 1970, 6, 76-90.

(30) Fletcher, R. A New Approach to Variable Metric. Algorithms. Comp. J. 1970, 13, 317-322;

(31) Goldfarb, D. A Family of Variable Metric Updates Derived by Variational Means. Math. Comp. 1970, 24, 23-26.

(32) Shanno, D. F. Conditioning of quasi-Newton methods for function minimization. Math. Comp. 1970, 24, 647-656.

(33) Gale, J. D. GULP: A computer program for the symmetry-adapted simulation of solids. J. Chem. Soc. Faraday Trans. 1997, 93, 629-637.

(34) Gale, J. D.; Rohl, A. L. The General Utility Lattice Program (GULP). Mol. Simul. 2003, 29, 291-341.

(35) Li, Y.; Yu, J.; Xu, R. Criteria for Zeolite Frameworks Realizable for Target Synthesis. Angew. Chem. Int. Ed. 2013, 52, 1673-1677.

(36) Liu, X; Valero, S.; Argente, E.; Botti, V.; Sastre, G. The importance of T...T..T angles in the feasibility of zeolites. Z. Kristallogr. 2015, 230, 291-299.

(37) Staples, L.W.; Gard, J.A. The fibrous zeolite erionite; its occurrence, unit cell, and structure. Mineral. Mag., 1959, 32, 261-281.

(38) Schlenker, J. L.; Pluth, J. J.; Smith, J. V. Dehydrated natural erionite with stacking faults of the offretite type. Acta Crystallogr., 1977, B33, 3265-3268.

(39) Lee, J. H.; Park, M. B.; Lee, J. K.; Min, H. K.; Song, M. K.; Hong, S. B. Synthesis and Characterization of ERI-Type UZM-12 Zeolites and Their Methanol-to-Olefin Performance. J. Am. Chem. Soc. 2010, 132, 12971-12982.

(40) Miller, M. A.; Lewis, G. J.; Gisselquist, J. L.; Moscoso, J. G.; Patton, R. L. UZM-12 and UZM-12HS: crystalline aluminosilicate zeolitic compositions and processes for preparing and using the compositions. U. S. Patent 7,344,694, 2008.

(41) Xe, D.; Zones, S. I.; Lew, C. M.; Davis, T. M. Molecular sieve SSZ-98. U. S. Patent 9,409,786, 2016.

(42) Boruntea, C.-R.; Lundegaard, L. F.; Corma, A.; Vennestrøm, P. N. R.; Crystallization of AEI and AFX zeolites through zeolite-to-zeolite transformations. Micropor. Mesopor. Mater. 2019, 278, 105-114.

(43) Teketel, S.; Lundegaard, L. F.; Skistad, W.; Chavan, S. M.; Olsbye, U.; Lillerud, K. P.; Beato, P.; Svelle, S. Morphology-induced shape selectivity in zeolite catalysis. J. Catal. 2015, 327, 22-32.

(44) Łukaszuk, K. A.; Rojo-Gama, D.; Øien-Ødegaard, S.; Lazzarini, A.; Berlier, G.; Bordiga, S.; Lillerud, K. P.; Olsbye, U.; Beato, P.; Lundegaard, L. F.; Svelle, S. Zeolite morphology and catalyst performance: conversion of methanol to hydrocarbons over offretite. Catal. Sci. Technol. 2017, 7, 5435-5447.

(45) Xe, D.; Chen, C. Y. Synthesis of aluminosilicate zeolite SSZ-98. U. S. Patent 20,160,375,428, 2016

(46) Lillerud, K. P.; Raeder, J. H. On the synthesis of erionite-offretite intergrowth zeolites. Zeolites, 1986, 6, 474-483.

(47) Kubota, Y.; Helmkamp, M. M.; Zones, S. I.; Davis, M. E. Properties of organic cations that lead to the structure-direction of high-silica molecular sieves. Microporous Mater. 1996, 6, 213-229. 